\title{
Flow, Wind, and Stochastic Connectivity Modeling Infectious Diseases
}

\author{
C. Udriste $\mathbb{D}^{1},{ }^{1,2}$ I. Tevy $\mathbb{D}^{2}{ }^{2}$ and A. S. Rasheed ${ }^{2,3}$ \\ ${ }^{1}$ Academy of Romanian Scientists, Ilfov 3, 050044 Bucharest, Romania \\ ${ }^{2}$ University Politehnica of Bucharest, Department of Mathematics \& Informatics, Splaiul Independentei 313, \\ 060042 Bucharest, Romania \\ ${ }^{3}$ Ministry of Higher Education and Scientific Research, Baghdad, Iraq \\ Correspondence should be addressed to C. Udriste; udriste@mathem.pub.ro
}

Received 29 June 2020; Accepted 18 June 2021; Published 30 June 2021

Academic Editor: Mondher Farza

Copyright (c) 2021 C. Udriste et al. This is an open access article distributed under the Creative Commons Attribution License, which permits unrestricted use, distribution, and reproduction in any medium, provided the original work is properly cited.

\begin{abstract}
We study in this paper the trends of the evolution of different infections using a SIR flow (first-order ODE system), completed by a differential inclusion, a geodesic motion in a gyroscopic field of forces, and a stochastic SIR perturbation of the flow (Itô ODE system). We are interested in mathematical analysis, bringing new results on studied evolutionary models: infection flow together with a differential inclusion, bounds of evolution, dual description of disease evolution, log-optimal and rapid path, epidemic wind (geometric dynamics), stochastic equations of evolution, and stochastic connectivity. We hope that the paper will be a guideline for strategizing optimal sociopolitical countermeasures to mitigate infectious diseases.
\end{abstract}

\section{Introduction}

All topics in this paper are based on dynamics induced by flows and differential inclusions, dynamical systems of geometric origin, nonholonomic dynamical systems, and stochastic differential equations. Their combination reflects the mathematical complexity of the studied problems.

The mathematical literature that helped us to do this study is classified as follows: stochastic modeling of geometric structures [1-4], infectious disease flow [5-7], differential inclusions [8], geometric dynamics on Riemannian manifolds $[9,10]$, nonholonomic optimization $[11,12]$, and nonholonomic spaces [13].

The original results can be summarized by properties of infectious disease flow, Maple simulations for COVID-19 in Romania, infectious disease differential inclusion, bounds of disease evolution, dual description of disease evolution, epidemic wind generated by the flow and the geometry of the space, and computation of optimal striking time for stochastic connectivity.

The Maple simulations for COVID-19, with Romanian data, are more suggestive than what could be done according to the model related to the works [3,5-7]. Our results on the asymptotic behavior of infectious disease flow and bounds of disease evolution via extremum problems are finer than those presented by M. W. Hirsch, S. Smale, R. L. Devaney in their book [6], Chapter 11. The Pfaff evolution, the epidemic wind, and finding the optimal striking time for stochastic connectivity via an extremum problem are totally original ideas, suggested by our recent papers. Particularly, the Pfaff evolution underlines that we can study the infectious disease system like a Carnot group.

Although the epidemic differential inclusion and epidemic wind appear to have been created ad hoc, it explains the pandemic spread in the sense that any two points on the globe can be joined by an epidemic trajectory. This is not true if we stop with the explanations only at the epidemic flow. The same idea is underlined by stochastic connectivity.

1.1. Disease Infection Data. The evolution of disease infections in each region has been modeled recently via a stochastic susceptible-infected-recovered (SSIR) model [3, 5-7] with the following data: 
Evolution parameter: $t$ is the daily-time parameter.

States: (1) $S(t)$ denotes the total susceptible population at time $t$; (2) $I(t)$ denotes the number of active infections at time $t$; (3) $R(t)$ denotes the total number of recoveries and deaths at time $t$; (4) $\mathrm{d} S(t), \mathrm{d} I(t)$, and $\mathrm{d} R(t)$ denote the change in the states at time $t ;(5) \mathrm{d} W_{t}$ is an incremental Wiener process (Brownian motion), which models the randomness in the evolution.

Constant parameters: (1) $\beta$ (measured by $\left.[\beta]=(\text { people } \times \text { time })^{-1}\right)$ is a constant denoting the growth rate, which factors the rise in the number of infections, due to interactions between susceptible and infected populations. This parameter is a lumped constant which is meant to account for (a) the population size, (b) reproduction number of infectious diseases, and (c) exposure factor (which depends on mobility, precautionary measures, etc.); (2) $\gamma$ (measured by $\left.[\gamma]=(\text { time })^{-1}\right)$ is the rate of outcomes, that is, the rate at which the infections are neutralized, which may be due to recovery or death. It is assumed that recovered persons would not spread the infections again (at least for a window of a month); (3) $\sigma$ is a parameter used to model the randomness in the evolution, which may cause local deviations from the typical (exponential) trends; (4) $P_{\text {total }}$ is the population of the region, and $S_{0}$ and $I_{0}$ are the initial number of susceptible individuals and active infections.

\section{Infectious Disease Flow}

The susceptible-infected-recovered (SIR) model, with three different states, was selected [3,5 -7] to describe the evolution of different infections in a region of the world. On $\mathbb{R}_{+}^{3}$, the infectious disease Cauchy problem is

$$
\begin{aligned}
\dot{S}(t) & =-\beta S(t) I(t), \\
\dot{I}(t) & =(\beta S(t)-\gamma) I(t), \\
\dot{R}(t) & =\gamma I(t), \\
S(0) & =S_{0}, \\
I(0) & =I_{0}, \\
R(0) & =R_{0}, \\
S_{0}+I_{0}+R_{0} & =P_{\text {total }} .
\end{aligned}
$$

Since the initial differential system is equivalent to the symmetric system

$$
\frac{\mathrm{d} S}{-\beta S}=\frac{\mathrm{d} I}{\beta S-\gamma}=\frac{\mathrm{d} R}{\gamma},
$$

automatically two first integrals $S e^{(\beta / \gamma) R}=c_{1}$ and $S+I+R=$ $c_{2}$ appear, and hence the general solution is $S e^{(\beta / \gamma) R}=c_{1}, S+$ $I+R=c_{2}$ (spiral curve in a plane). In other words, the infectious disease nonlinear differential system is compartmental and proves the mass conservation property [5-7]. The family of field surfaces has the general equation $\phi\left(S e^{(\beta / \gamma) R}, S+I+R\right)=c$, where $\phi\left(c_{1}, c_{2}\right)$ is an arbitrary $C^{1}$ function. The vortex lines of a SIR vector field are plane curves.

Given an initial condition $S(0), I(0), R(0)$ summing to $P_{\text {total }}$, it follows $c_{1}=S_{0} e^{(\beta / \gamma) R_{0}}$ and $c_{2}=P_{\text {total }}=S_{0}+I_{0}+R_{0}$. Also, the previous differential system has a straight line of equilibrium points $I=0, S+R=P_{\text {total }} \quad$ (particularly, $S=0, I=0, R=P_{\text {total }}$ and $S=(\gamma / \beta), I=0, R=P_{\text {total }}-(\gamma / \beta)$ are two equilibrium points). The general theory shows that a nonisolated equilibrium point can be stable but not asymptotically stable.

\subsection{Open Problems}

(1) Investigate whether there are monomial connections $\nabla$ on $\mathbb{R}_{++}^{3}$ of components

$$
\Gamma_{i j}^{k}=c_{i j}^{k}\left(x^{1}\right)^{a_{1}}\left(x^{2}\right)^{a_{2}}\left(x^{3}\right)^{a_{3}}, \quad c_{i j}^{k}>0, x^{i} \in \mathbb{R}_{++}, a_{i} \in \mathbb{R},
$$

so that the SIR vector field is convex with respect to $\nabla$.

(2) Convolution is a very powerful technique in applications. Transforming the usual product into a convolution (product), let us replace the initial Cauchy problem with a convolution problem

$$
\begin{aligned}
\dot{S}(t) & =-\beta S(t) * I(t), \\
\dot{I}(t) & =(\beta S(t)-\gamma) * I(t), \\
\dot{R}(t) & =\gamma I(t), \\
S(0) & =S_{0}, \\
I(0) & =I_{0}, \\
R(0) & =R_{0}, \\
S_{0}+I_{0}+R_{0} & =P_{\text {total }},
\end{aligned}
$$

where the convolution (product) is defined by $(f * g)(t)=\int_{0}^{t} f(\tau) g(t-\tau) \mathrm{d} \tau$. Applying the Laplace transform, study the solution of this convolution problem.

2.2. Maple Simulations for COVID-19. We denote $S=x, I=y, R=z, t=$ daysassume values $\beta=7.5 \times 10^{-3}$ and $\gamma=0.06$ (hypothetical data used for research purpose). Romanian media, 06.05.2020: $P_{\text {total }}=$ Romania population $=19,410$ million; $x 0=$ quarantined + nonquarantined $=19,389 ; \quad y 0=$ infected $=13,837 ; \quad z 0=$ recovered $5.454+$ deceased $858=6,312$ (real data normalized by 1,000).

The graph $t, x(t)$ admits a limit point $x=\bar{x}$ $=\lim _{t \rightarrow \infty} x(t) \geq 0$; the graph $t, y(t)$ has a maximum point and a limit point $y=\bar{y}=\lim _{t \rightarrow \infty} y(t)=0$. The graph $t, z(t)$ has a limit point $z=\bar{z}=\lim _{t \longrightarrow \infty} z(t) \geq 0$. Of course, $\bar{x}+\bar{z}=P_{\text {total }}$. See Figures $1-3$.

(i) with(DEtools): 


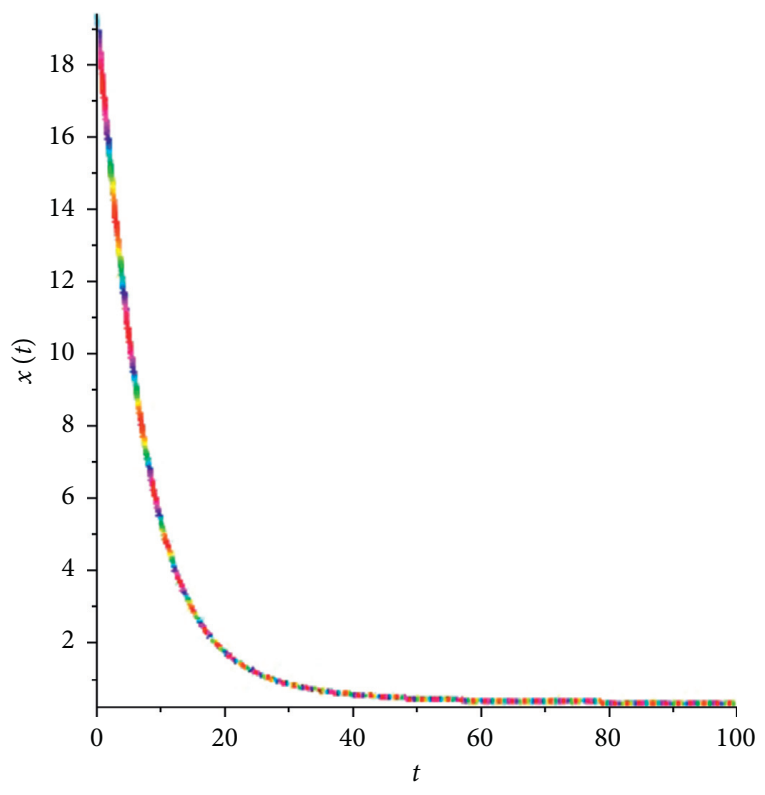

FigURE 1: Susceptible population evolution $t, x(t)$.

phaseportrait $\left(\left[D(x)(t)=-7.5 * 10^{(-3)} * x(t) * y\right.\right.$ $(t),(D(y))(t)=\left(7.5 * 10^{(-3)} * x(t)-0.6 e-1\right) *$ $y(t),(D(t))(t)=0.6 e-1 * y(t)],[x(t), y(t), z(t)]$, $t=0 \quad . .100,[[x(0)=19.389, y(0)=13.837, z(0)=$ 6.312] $], \quad$ stepsize $=0.5 e-1, \quad$ scene $=[t, \quad x(t)]$, linecolour $=\sin ((1 / 2) * t * P i)$, method $=$ classical [foreuler]);

(ii) phaseportrait $\left(\left[D(x)(t)=-7.5 * 10^{(-3)} * x(t) * y\right.\right.$ $(t),(D(y))(t)=\left(7.5 * 10^{(-3)} * x(t)-0.6 e-1\right) *$ $y(t),(D(t))(t)=0.6 e-1 * y(t)],[x(t), y(t), z(t)]$, $t=0, \ldots, 100,[[x(0)=19.389, y(0)=13.837, z(0)=$ 6.312] $], \quad$ stepsize $=0.5 e-1, \quad$ scene $=[\mathrm{t}, \quad y(t)]$, linecolour $=\sin ((1 / 2) * t * P i))$, method $=$ classical [foreuler]);

(iii) phaseportrait $\left(\left[D(x)(t)=-7.5 * 10^{(-3)} * x(t) * y\right.\right.$ $(t),(D(y))(t)=\left(7.5 * 10^{(-3)} * x(t)-0.6 e-1\right) *$ $y(t),(D(t))(t)=0.6 e-1 * y(t)],[x(t), y(t), z(t)]$, $t=0, \ldots, 100,[[x(0)=19.389, y(0)=13.837, z(0)=$ 6.312]], stepsize $=0.5 e-1, \quad$ scene $=[t, \quad z(t)]$, linecolour $=\sin ((1 / 2) * t * P i))$, method $=$ classical [foreuler]).

Remark 1. Let us animate the surface $S e^{(\beta / \gamma) R}=c_{1}$ with respect to the parameter $a=(\beta / \gamma)$ (Figure 4).

(i) with(plots);

(ii) animate (plot3d, $[x * \exp (a * z), x=1, \ldots, 10, z=1, \ldots, 10]$, $a=0.125, \ldots, 1)$.

2.3. Parametrization by $S$. It would be more natural to parameterize the previous general solution by "raw material" S; namely,

$$
\begin{aligned}
S & =S, \\
I & =\frac{\gamma}{\beta} \ln \left(c_{2} S\right)-S, \\
R & =-\frac{\gamma}{\beta} \ln \left(c_{1} S\right),
\end{aligned}
$$

where $c_{1} S<1<c_{2} S$ and $(\gamma / \beta) \ln \left(c_{2} / c_{1}\right)=P_{\text {total }}$. If the social constants $\beta, \gamma$ and the state $S$ are so that $I>0$, then there are infections; otherwise, there are not. So, trying to influence the transmission constants $\beta, \gamma$ we can limit the number of infections. In fact, the state $I$ exists, if and only if $S_{1} \leq S \leq S_{2}$ and then $I\left(S_{1}\right)=I\left(S_{2}\right)=0$.

If we start with the equilibrium points $S_{0}=P_{\text {total }}, I_{0}=0$, and $R_{0}=0$, we find

$$
\begin{aligned}
& I=\frac{\gamma}{\beta} \ln \left(\frac{S}{S_{0}}\right)+S_{0}-S, \\
& R=-\frac{\gamma}{\beta} \ln \left(\frac{S}{S_{0}}\right), \quad S<S_{0} .
\end{aligned}
$$

The more interesting case holds for $S_{0}>(\gamma / \beta)$. Then, the ODE for $S(t)$ becomes

$$
\dot{S}(t)=\beta S(t)^{2}-\beta S_{0} S(t)-\gamma S(t) \ln \left(\frac{S(t)}{S_{0}}\right),
$$

or changing the variable as $S(t)=S_{0} u(t)$,

$$
\dot{u}(t)=\beta S_{0}\left(u(t)^{2}-u(t)\right)-\gamma u(t) \ln u(t), \quad u(t)<1 .
$$

The solution of this ODE is written in the form

$$
t+c=\int \frac{\mathrm{d} u}{\beta S_{0}\left(u^{2}-u\right)-\gamma u \ln u} .
$$

The denominator of the integrand has three roots $0, u_{0}$, and 1 . Using the qualitative approximation

$$
t+c=k \int \frac{\mathrm{d} u}{u\left(u-u_{0}\right)(u-1)}, \quad u_{0}<u<1,
$$

we find the implicit solution

$$
\frac{u^{1 / u_{0}}(1-u)^{1 /\left(1-u_{0}\right)}}{\left(u-u_{0}\right)^{1 / u_{0}\left(1-u_{0}\right)}}=c e^{t / k} .
$$

The constant $c$ is determined from the initial condition $u(0)=1-\varepsilon$. The initial condition $u(0)=1$ would give us the equilibrium point $u(t) \equiv 1$.

Let us observe that $u(t) \longrightarrow u_{0}$ for $t \longrightarrow \infty$. Hence, the final state $I=0, S=S_{1}$ appears for $t \longrightarrow \infty$.

We fix $\beta=1, \gamma=3, S_{0}=5$. From the graphs $S, I(S)$ and $\left(S, F(S)=S^{2}-5 S-3 S \ln (S / 5)\right)$, in Figure 5 , we can read the following: when $S$ is decreasing from $S_{0}$ to $S_{1}$, the function $I$ is increasing from 0 to a maximum for $S=\beta / \gamma$ and then decreasing again to 0 . In other words, when the epidemic goes out, that is, $I=0$ again, and the variable $S$ stops at $S_{1}>0$, then we talk about an uncontaminated population. 


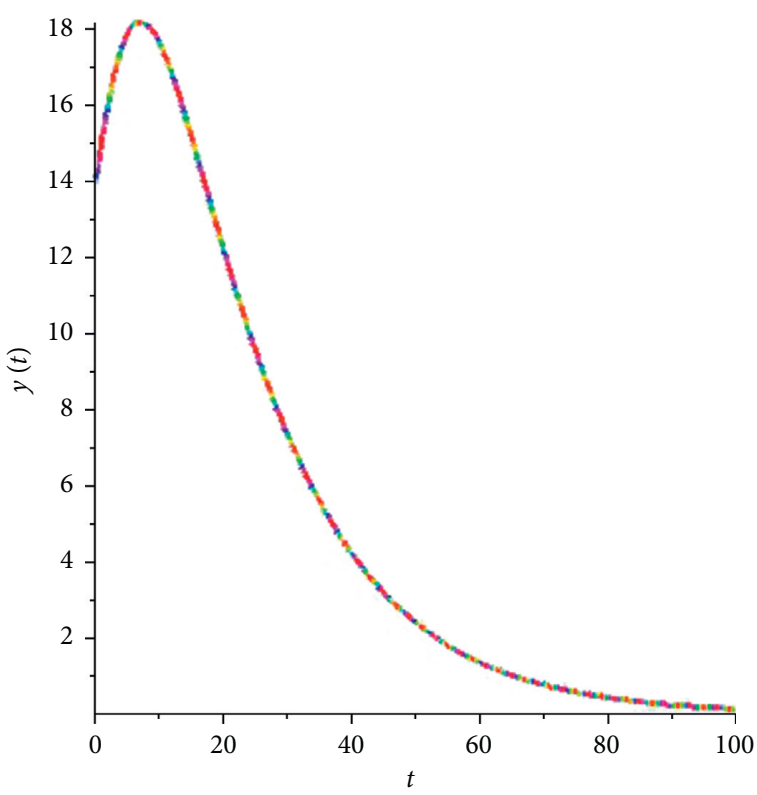

Figure 2: Active infection evolution $t, y(t)$.

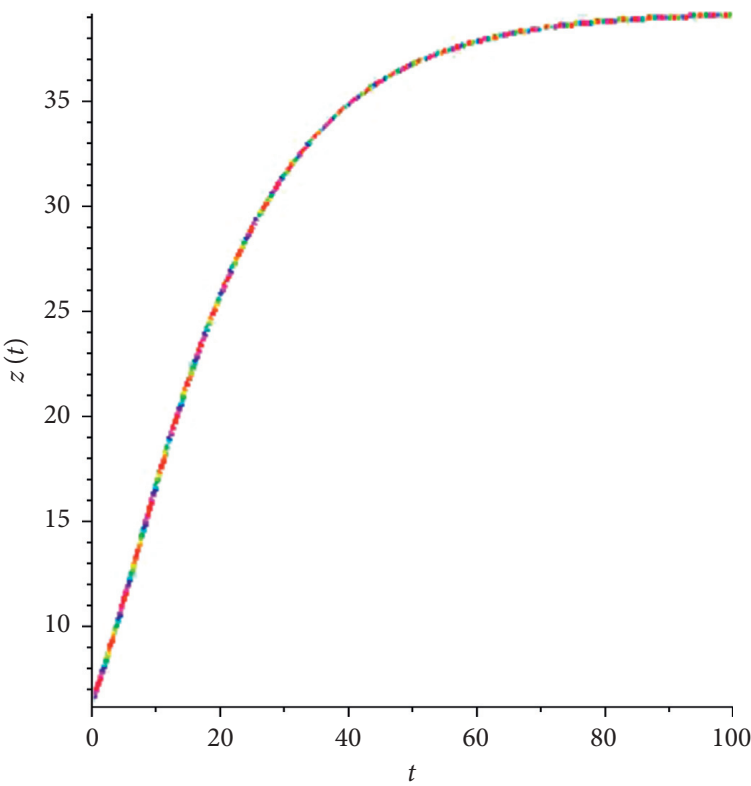

Figure 3: Number of recoveries and deaths' evolution $t, z(t)$.

Maple simulation (Figure 5):

(i) $\operatorname{plot}\left(\left[3 * \ln ((1 / 5) * x)+5-x, x^{2}-5 * x-3 * x *\right.\right.$ $\ln ((1 / 5) * x)], x=0 . .6$, color $=[$ "red", "green"] $]$.

\subsection{Asymptotic Behavior of Infectious Disease Flow}

Theorem 1. For positive initial conditions $\left(S_{0}, I_{0}, R_{0}\right)$, the limit value $\bar{S}+\bar{I}=\lim _{t \rightarrow \infty}(S(t)+I(t))$ exists and is $P_{\text {total }}-\bar{R}$.
Proof. Suppose $S(t) \leq(\gamma / \beta)$

Because $S(t)$ is monotonically decreasing $(\dot{S}(t)=-\beta S(t) I(t) \leq 0)$ and nonnegative, it has a limit $\bar{S}=\lim _{t \rightarrow \infty} S(t)$, with $0 \leq \bar{S} \leq(\gamma / \beta)$.

Since $I(t)$ is monotonically decreasing $(\dot{I}(t)=(\beta S(t)-\gamma) I(t) \leq 0)$ and nonnegative, it has a limit $\bar{I}=\lim _{t \rightarrow \infty} I(t)$, with $\bar{I} \geq 0$.

On the other hand, $R(t)$ is monotonically increasing $(\dot{R}(t)=\gamma I(t) \geq 0)$ and nonnegative. But $S(t)+I(t)+R(t)=$ $P_{\text {total }}$ shows that $R(t)$ has a limit $\bar{R} \geq 0$. For $t$ large enough, we have $S(t) \approx \bar{S}, I(t) \approx \bar{I}$, and $R(t) \approx \bar{R}$. Then, the differential 


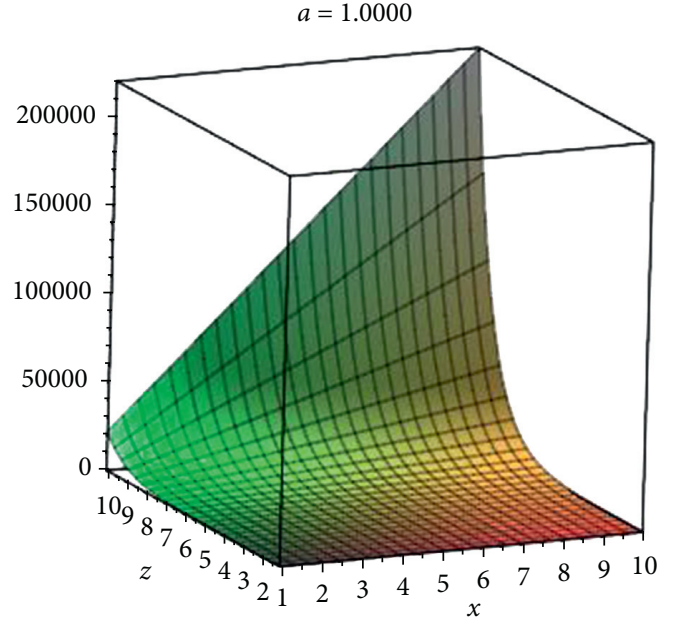

Animate SR first integral

FIgURE 4: Animate SR first integral.

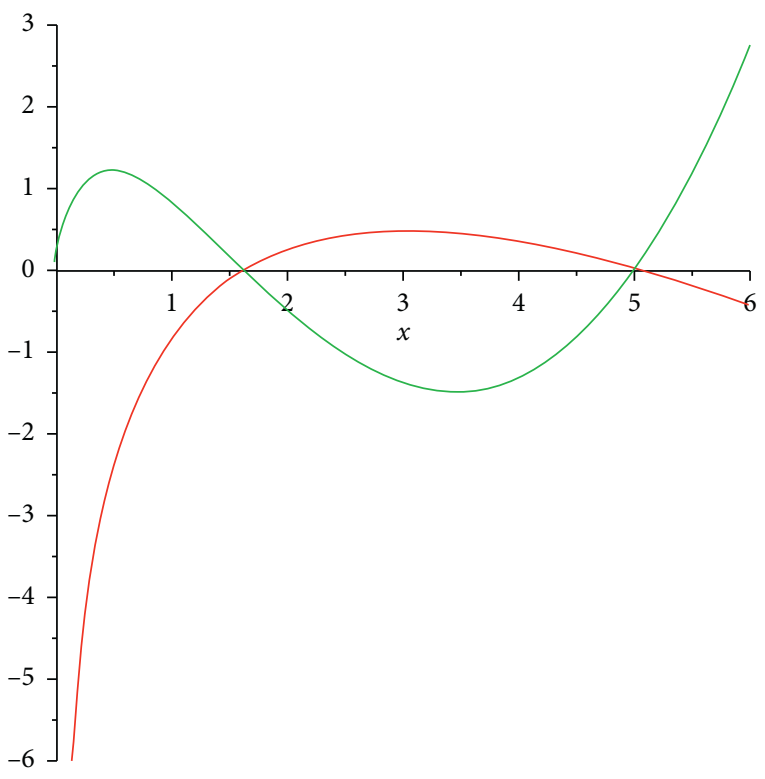

Figure 5: Intersection $I(S) \cap F(S)$.

system consisting of the first equation and third equation converges to the linear system corresponding to the linearization at the point $(\bar{S}, \bar{I})$; that is,

$$
\begin{aligned}
\dot{S} & =-\beta \bar{I}(S(t)-\bar{S})-\beta \bar{S}(I(t)-\bar{I}), \\
\dot{R}(t) & =\gamma \bar{I}(I(t)-\bar{I}) .
\end{aligned}
$$

The matrix of this linear system has the eigenvalues $\lambda_{1}=$ $-\beta \bar{I}<0$ and $\lambda_{2}=\gamma \bar{I}>0$. Consequently, $(\bar{S}, \bar{I})$ render this system unstable, and then the trajectory $S(t), I(t), R(t)$ diverges. This contradicts $S(t)+I(t)+R(t)=P_{\text {total }}$.

Suppose $S(t)>(\gamma / \beta)$. Similarly, it follows the existence of $(\bar{S}, \bar{I}, \bar{R})$, satisfying $(\gamma / \beta) \leq \bar{S}, \bar{I} \geq 0$, and $\bar{R} \geq 0$.

Theorem 2. For the infectious disease flow, one has $\bar{I}=\lim _{t \longrightarrow \infty} I(t)=0$ (see equilibrium point).
Proof. We use the second differential equation $\mathrm{d} I(t) / I(t)=(\mathrm{d} / \mathrm{d} t) \ln I(t)=\beta S(t)-\gamma$. Let $\delta$ be the primitive of function $S(t)$. Since $\int_{0}^{b} \beta S(t)-\gamma \mathrm{d} t=\ln (I(b) / I(0))$, we find $\beta(\delta(b)-\delta(0))-\gamma b=\ln (I(b) / I(0))$. At limit, when $b \longrightarrow \infty$, we must have $\bar{I}=I(\infty)=0$ (see equilibrium point).

Remark 2. Let $\mathcal{S}, \mathscr{I}$, and $\mathscr{R}$ be the primitives of functions $S(t), I(t)$, and $R(t)$. Then, $\mathcal{S}(t)+\mathscr{I}(t)+\mathscr{R}(t)=t P_{\text {total }}$.

Theorem 3. Let $\mathcal{S}, \mathscr{I}$, and $\mathscr{R}$ be the primitives of functions $S(t), \quad I(t)$, and $R(t)$. For positive initial conditions $\left(S_{0}, I_{0}, R_{0}\right)$, the limit values

$$
\begin{aligned}
& \bar{S}=\lim _{t \longrightarrow \infty} S(t), \\
& \overline{\mathscr{I}}=\lim _{t \longrightarrow \infty} \mathscr{I}(t), \\
& \bar{R}=\lim _{t \longrightarrow \infty} R(t)
\end{aligned}
$$

are related by

$$
\begin{aligned}
-\beta(\overline{\mathscr{I}}-\mathscr{I}(0)) & =\ln \frac{\bar{S}}{S(0)}, \\
\bar{R}-R(0) & =\gamma(\overline{\mathscr{I}}-\mathscr{I}(0)) .
\end{aligned}
$$

Proof. Let us consider the first differential equation

$$
\frac{\mathrm{d} S(t)}{S(t)}=\frac{\mathrm{d}}{\mathrm{d} t} \ln S(t)=-\beta I(t) \mathrm{d} t .
$$

It follows $-\beta \int_{0}^{\infty} I(t) \mathrm{d} t=\ln (\bar{S} / S(0))$, and hence $-\beta(\overline{\mathscr{I}}-\mathscr{I}(0))=\ln (\bar{S} / S(0))$.

Integrating the third differential equation, we find

$$
\bar{R}-R(0)=\gamma(\overline{\mathscr{I}}-\mathscr{I}(0)) .
$$

Since $(S, I, R)$ represent the evolution of disease infections, the initial conditions $S_{0} \geq 0, I_{0} \geq 0, R_{0} \geq 0$, and $S_{0}+I_{0}+R_{0}=P_{\text {total }}$ are perfectly suited for biological applications. Suppose these initial conditions. The monotony of the state functions $S(t), I(t), R(t)$ is described by the signum of derivatives: either $\dot{S} \leq 0, \dot{I}>0, \dot{R} \geq 0$ which show that $S(t)$ is decreasing and $I(t)$ and $R(t)$ are increasing $\forall t>0$ or $\dot{S} \leq 0, \dot{I} \leq 0, \dot{R} \geq 0$ which show that $S(t), I(t)$ are decreasing and $R(t)$ is increasing $\forall t>0$.

2.5. Covering All the Manifold by a Differential Inclusion. As any flow, the infectious disease flow does not cover all the manifold $\mathbb{R}_{+}^{3}$, and so there are pairs of points that cannot be joined by a flow trajectory. The natural question arises: what mathematical construction allows us to cover all the manifold?

The infectious disease vector field $X=I(-\beta S, \beta S-\gamma, \gamma)$ determines an orthogonal distribution generated by two linearly independent vector fields $Y=(\beta S-\gamma, \beta S, 0)$ and $Z=(0,-\gamma, \beta S-\gamma)$, orthogonal to $X$.

The three vector fields $X, Y, Z$ determine the differential inclusion 


$$
\frac{\mathrm{d}}{\mathrm{d} t}\left(\begin{array}{c}
S \\
I \\
R
\end{array}\right) \in\{X, S p\{Y, Z\}\},
$$

which can be used to understand and suitably interpret the spreading of the disease, in the sense that any two points on $\mathbb{R}_{+}^{3}$ can be joined by a piecewise solution of the differential inclusion.

\section{Bounds of Disease Evolution}

Let us select the best values of state variables when we evolve along the solution of the disease flow. The aim is to manage correctly a pandemic since the values of certain parameters can be chosen subject to some conditions expressing their ranges and interrelationships. The choice determines the values of a number of other variables on which the desirability of the end result depends, such as cost, weight, speed, bandwidth, and reliability.

3.1. Extrema Constrained by Equalities. A basic problem we discuss at the beginning of this paper is as follows: "find $\max f(S, I, R)=I$ subject to $S e^{(\beta / \gamma) R}=c_{1}, S+I+R=c_{2}$."

The constraints satisfy the condition of nondegenerate constraint qualification.

To solve this problem, we attach the Lagrange function

$$
F=I+\lambda\left(S e^{(\beta / \gamma) R}-c_{1}\right)+\mu\left(S+I+R-c_{2}\right) .
$$

Since

$$
\mathrm{d} F=\mathrm{d} I+\lambda\left(e^{(\beta / \gamma) R} \mathrm{~d} S+S \frac{\beta}{\gamma} e^{(\beta / \gamma) R} \mathrm{~d} R\right)+\mu(\mathrm{d} S+\mathrm{d} I+\mathrm{d} R)
$$

the critical points of the function $F$ are given by the algebraic system

$$
\begin{aligned}
\frac{\partial F}{\partial S} & =\lambda e^{(\beta / \gamma) R}+\mu=0, \\
\frac{\partial F}{\partial I} & =1+\mu=0, \\
\frac{\partial F}{\partial R} & =\lambda S \frac{\beta}{\gamma} e^{(\beta / \gamma) R}+\mu=0, \\
S e^{(\beta / \gamma) R} & =c_{1}, \\
S+I+R & =c_{2} .
\end{aligned}
$$

It follows the critical points

$$
\begin{aligned}
& S=\frac{\gamma}{\beta}, \\
& R=-\frac{\gamma}{\beta} \ln \lambda, \\
& I=c_{2}-\frac{\gamma}{\beta}-\frac{\gamma}{\beta} \ln \lambda, \\
& \lambda=\frac{\gamma}{\beta c_{1}}, \\
& \mu=-1 .
\end{aligned}
$$

On the other hand,

$$
\mathrm{d}^{2} F=\lambda\left(2 \frac{\beta}{\gamma} e^{(\beta / \gamma) R} \mathrm{~d} S \mathrm{~d} R+S\left(\frac{\beta}{\gamma}\right)^{2} e^{(\beta / \gamma) R} \mathrm{~d} R^{2}\right) .
$$

The associated matrix

$$
\left(\begin{array}{cc}
0 & \lambda \frac{\beta}{\gamma} e^{(\beta / \gamma) R} \\
\lambda \frac{\beta}{\gamma} e^{(\beta / \gamma) R} & \lambda S\left(\frac{\beta}{\gamma}\right)^{2} e^{(\beta / \gamma) R}
\end{array}\right)
$$

with respect to $\{\mathrm{d} S, \mathrm{~d} R\}$, is negative definite. The function $F$ is concave. Hence, all critical points are maximum points. The maximum value of objective function $I$ is

$$
I_{\max }=c_{2}-\frac{\gamma}{\beta}-\frac{\gamma}{\beta} \ln \lambda
$$

The introduction of Lagrange multipliers $\lambda, \mu$ as additional variables looks artificial but it makes it possible to apply to the constrained-extremum problem the same firstorder condition used in the free-extremum problem (but for more complex function $F$ ). Note also that $\lambda, \mu$ have a certain specific meaning: if the solution is regarded as

$$
S^{*}\left(c_{1}, c_{2}\right), I^{*}\left(c_{1}, c_{2}\right), R^{*}\left(c_{1}, c_{2}\right), \lambda^{*}\left(c_{1}, c_{2}\right), \mu^{*}\left(c_{1}, c_{2}\right),
$$

then the marginal variations are

$$
\begin{aligned}
& \frac{\partial}{\partial c_{1}} I\left(S^{*}\left(c_{1}, c_{2}\right), I^{*}\left(c_{1}, c_{2}\right), R^{*}\left(c_{1}, c_{2}\right)\right)=\lambda^{*}\left(c_{1}, c_{2}\right), \\
& \frac{\partial}{\partial c_{2}} I\left(S^{*}\left(c_{1}, c_{2}\right), I^{*}\left(c_{1}, c_{2}\right), R^{*}\left(c_{1}, c_{2}\right)\right)=\mu^{*}\left(c_{1}, c_{2}\right) .
\end{aligned}
$$

Maple Simulations: we denote $S=x, I=y, R=z$. Let us find the extrema of some functions constrained by the spiral curve.

(i) with (optimization);

The Minimize command automatically selects the most appropriate solver. 
(ii) $\operatorname{Minimize}(x, \quad x * \exp (z)=1, x+y+z=2$, assu$\mathrm{me}=$ nonnegative $) ; \quad 0.158594339562640796,[x=$ HFloat (0.1585943395626408), $y=\operatorname{HFloat}(0.0), z$ $=$ HFloat $(1.8414056604373585)]$

(iii) $\operatorname{Minimize}(y, \quad x * \exp (z)=10, x+y+z=50$, assume $=$ nonnegative $) ; \quad 39.9999999999999858$, [ $x=$ HFloat $(10.0), y=$ HFloat $(39.9999999999999$ 86), $z=$ HFloat (0.0)]

(iv) $\operatorname{Minimize}(z, \quad x * \exp (z)=10, x+y+z=50$, assume $=$ nonnegative $) ; \quad 0 .,[x=\operatorname{HFloat}(1.0), y$ $=$ HFloat $(0.9999999999999998), z=\operatorname{HFloat}(0.0)]$

(v) $\operatorname{Minimize}(y, \quad x * \exp (z)=a, x+y+z=b$, assume $=$ nonnegative $) ; \quad 0 .,[a=\operatorname{HFloat}(1.1097167$ 915216666), $b=$ HFloat (1.2345190270116868), $x=$ HFloat (0.5722702360103161), $y=$ HFloat $(0.0), z$ $=$ HFloat $(0.6622487910013707)]$

(vi) $\operatorname{Minimize}(y, \quad x * \exp (10 * z)=a, x+y+z=b$, assume $=$ nonnegative $) ; \quad 0 .,[a=$ HFloat $(1.4613$ 527630961411), $b=$ HFloat (1.458647134440591), $x=$ HFloat (1.4584481765262327),$y=$ HFloat (0.0), $z$ = HFloat (1.9895791435813165e-4)]

(vii) $\operatorname{Minimize}\left(x^{2}+y^{2}+z^{2}, x * \exp (10 * z)=1, x+y\right.$ $+z=2$, assume $=$ nonnegative); 1.9941556847397 1461, [ $x=$ HFloat0.9413968463983702 $y=$ HFloat $1.052564103608946, z=$ HFloat 0.00603904999268 35095]

3.2. Extrema Constrained by Field Lines. To simplify, we use standard notations in mathematics $S=x^{1}, I=x^{2}, R=x^{3}$. Then, infectious disease Cauchy problem on $\mathbb{R}_{+}^{3}$ is written

$$
\begin{aligned}
\dot{x}^{1}(t) & =-\beta x^{1}(t) x^{2}(t), \\
\dot{x}^{2}(t) & =\left(\beta x^{1}(t)-\gamma\right) x^{2}(t), \\
\dot{x}^{3}(t) & =\gamma x^{2}(t), \\
x^{1}(0) & =x_{0}^{1}, \\
x^{2}(0) & =x_{0}^{2}, \\
x^{3}(0) & =x_{0}^{3}, \\
x_{0}^{1}+x_{0}^{2}+x_{0}^{3} & =P_{\text {total }} .
\end{aligned}
$$

To find bounds of significant functions connected to this flow, we use the technique of optimization of an objective function constrained by a field line $x=x t, x_{0}, t \in 0, \infty, x_{0}=x_{0}^{1}, x_{0}^{2}, x_{0}^{3}$.

Let us start with finding the maximum for the total susceptible population $x^{1}(t)$ : findmax $f\left(x^{1}, x^{2}, x^{3}\right)=x^{1}$ with the restriction $x=x\left(t, x_{0}\right)$.

Since the component $x^{1}(t)$ is a decreasing function, the maximum is reached at the starting point $x_{0}$.

Let us show that we do not have constrained critical points that produce an extremum. We set the critical point condition $\langle\nabla f, X\rangle=0$. In this case, $\nabla f=(1,0,0)$ and hence $-\beta x^{1}(t) x^{2}(t)=0$.

We eliminate $x^{2}=0$, since this condition leads to an equilibrium point of the dynamical system. Also, the solution $x^{1}=0$ is not convenient. Indeed, a critical point of the form $\bar{x}=\left(\bar{x}^{1}=0, \bar{x}^{2}>0, \bar{x}^{3} \geq 0\right)$ cancels the expression Hess $f(X, X)(\bar{x})+\left\langle\nabla f, D_{X} X\right\rangle(\bar{x})$ whose sign at the point $\bar{x}$ would decide the property of extremum: $\left\langle\nabla f, D_{X} X\right\rangle(\bar{x}) \equiv 0$, that is, $\beta x^{1} x^{2}-\beta^{2}\left(x^{1}\right)^{2}+\beta \gamma x^{1} \equiv 0$.

Since $\quad \ddot{x}^{1}(t)=\beta x^{1}(t) x^{2}(t)\left(\beta x^{2}(t)-x^{1}(t)+\gamma\right)$, the function $x^{1}(t)$ is convex on the subset $\beta x^{2}(t)-x^{1}(t)+\gamma \geq 0$.

Proposition 1. The maximum of the total susceptible population is reached at the starting point $x_{0}$.

Let us find now the maximum for the number of active infections $x^{2}(t)$ : determine $\max f\left(x^{1}, x^{2}, x^{3}\right)=x^{2}$ constrained by $x=x\left(t, x_{0}\right)$.

We set the critical point condition $\langle\nabla f, X\rangle=0$. In this case, $\quad \nabla f=(0,1,0)$. It follows the relation $\beta x^{1}(t)-\gamma x^{2}(t)=0$. The convenient solution (critical point) is $\bar{x}=\left(\bar{x}^{1}=(\gamma / \beta) \geq 0, \bar{x}^{2}>0, \bar{x}^{3} \geq 0\right)$.

The sufficient condition Hess $f(X, X)(\bar{x})+\langle\nabla f$, $\left.D_{X} X\right\rangle(\bar{x})<0$ is equivalent to $\left\langle\nabla f, D_{X} X\right\rangle(\bar{x})<0$ or $-\beta^{2} \bar{x}^{1} \bar{x}^{2}+\left(\beta \bar{x}^{1}-\gamma\right)^{2}<0$.

Theorem 4. Suppose that on an evolution field line there exists a point $\bar{x}$ satisfying

$$
\left(\frac{\beta^{2}}{\gamma^{2}}-1\right)^{2}<\frac{\beta^{3}}{\gamma^{3}} \bar{x}^{2} .
$$

Then, the number of active infections has an upper bound at this point.

To find the minimum for the number of recoveries and deaths $x^{3}(t)$, we use the problem: determine $\min f\left(x^{1}, x^{2}, x^{3}\right)=x^{3}$ constrained by $x=x\left(t, x_{0}\right)$.

Since the function $x^{3}(t)$ is increasing, the minimum is reached at the starting point $x_{0}$.

Let us show that we do not have constrained critical points that produce an extremum. We set the critical point condition $\langle\nabla f, X\rangle=0$. In this case, $\nabla f=(0,0,1)$. It follows the relation $\gamma x^{2}(t)=0$. We have no convenient solution (critical point) since $x^{2}=0$ leads to an equilibrium point of the dynamical system.

On the subset $x^{1} \geq(\gamma / \beta)$, the function $x^{3}(t)$ is convex. Indeed, we have $\ddot{x}^{3}(t)=\gamma\left(\beta x^{1}(t)-\gamma\right) x^{2}(t)$.

Proposition 2. The minimum number of recoveries and deaths is reached at the starting point $x_{0}$.

\section{Dual Description of Disease Evolution}

A vector field determines a flow (collinearity condition) and a Pfaff equation (orthogonality condition) [9]. By duality, the nonlinear ODEs in infectious disease flow are transformed into an infectious disease Pfaff equation

$$
\omega=-\beta S I \mathrm{~d} S+(\beta S-\gamma) I \mathrm{~d} I+\gamma I \mathrm{~d} R=0 .
$$


Since $\omega \wedge \mathrm{d} \omega=\beta \gamma I^{2} \mathrm{~d} S \wedge \mathrm{d} I \wedge \mathrm{d} R$, this Pfaff equation is noncompletely integrable (it represents a nonholonomic surface, i.e., a collection of integral curves). The integral curves are orthogonal to infectious disease field lines. Since any two points in $\mathbb{R}_{+}^{3}$ are joined by an integral curve of this Pfaff equation, the dual evolution of infectious disease shows that all parts of the world will be infected.

Simplifying by $I$, we obtain an equivalent infectious disease Pfaff equation

$$
\beta S \mathrm{~d} S-(\beta S-\gamma) \mathrm{d} I-\gamma \mathrm{d} R=0 .
$$

The normal vector field to this distribution is $N=(\beta S,-(\beta S-\gamma),-\gamma)$. Two independent vector fields tangent to the distribution are

$$
\begin{aligned}
& X=\frac{\partial}{\partial S}+\frac{\beta S}{\gamma} \frac{\partial}{\partial R}, \\
& Y=\frac{\partial}{\partial I}-\left(\frac{\beta S-\gamma}{\gamma}\right) \frac{\partial}{\partial R} .
\end{aligned}
$$

It follows

$$
\begin{aligned}
& {[X, Y]=Z=-\frac{\beta}{\gamma} \frac{\partial}{\partial R},} \\
& {[X, Z]=0,} \\
& {[Y, Z]=0,}
\end{aligned}
$$

and hence the vector fields $X, Y, Z$ determine a Carnot group (in a future paper we shall study the infectious disease system like a Carnot group).

Proposition 3. For $t \in[0, b]$, one has

$$
\beta S^{2}(b)+2 \gamma R(b) \geq \beta S^{2}(0)+2 \gamma R(0) .
$$

Proof. For $t \in[0, b]$, we consider the curve $c(t)=S(t), I(t), R(t)$. Integrating the Pfaff form and selecting $I(t)$ in a convenient way, it follows

$$
\begin{aligned}
\frac{\beta}{2}\left(S^{2}(b)-S^{2}(0)\right)+\gamma(R(b)-R(0)) & =\int_{c(t)}(\beta S-\gamma) \mathrm{d} I=\int_{0}^{b} \beta S(t)-\gamma \dot{I}(t) \mathrm{d} t \\
& =\int_{0}^{b} \beta S(t)-\gamma^{2} I(t) \mathrm{d} t \geq 0 .
\end{aligned}
$$

4.1. Log-Optimal and Rapid Path. To find bounds of significant functions for disease distribution, we can use the technique of optimization of an objective function with nonholonomic constraints $[11,12]$. One of these functions is the "volume" (SIR product) of disease states.

To find bounds for the function $g(S, I, R)=\ln |\operatorname{SIR}|$ (logarithm of "volume"), we use the problem "determine $\max g(S, I, R)$ constrained by the Pfaff equation of evolution."

The critical point condition is

$$
\eta=\frac{\mathrm{d} S}{S}+\frac{\mathrm{d} I}{I}+\frac{\mathrm{d} R}{R}+\lambda(-\beta S I \mathrm{~d} S+(\beta S I-\gamma I) \mathrm{d} I+\gamma I \mathrm{~d} R) \equiv 0 .
$$

It follows

$$
\begin{aligned}
\lambda(\beta S I) & =\frac{1}{S}, \\
\lambda(\beta S I-\gamma I) & =-\frac{1}{I}, \\
\lambda(\gamma I) & =-\frac{1}{R},
\end{aligned}
$$

or

$$
\lambda=\frac{1}{S^{2} I}=-\frac{1}{(\beta S-\gamma) I^{2}}=-\frac{1}{\gamma R I} .
$$

Consequently, the critical point set is described by

$$
\begin{aligned}
(\beta S-\gamma) I & =S^{2}, \\
S^{2} & =\gamma R, \\
S+I+R & =P_{\text {total }},
\end{aligned}
$$

that is,

$$
\begin{aligned}
S & =\frac{1}{2}\left(\beta I+\sqrt{\left(\beta^{2} I-4 \gamma\right) I}\right), \\
R & =\left(\frac{\beta}{\gamma} S-1\right) I, \\
S+I+R & =P_{\text {total }},
\end{aligned}
$$

subject to the condition $\beta^{2} I-4 \gamma \geq 0$. This algebraic system has a solution since the curvel

$$
\begin{aligned}
S & =\frac{1}{2}\left(\beta I+\sqrt{\left(\beta^{2} I-4 \gamma\right) I}\right), \\
I & =I, \\
R & =\left(\frac{\beta}{\gamma} S-1\right) I,
\end{aligned}
$$

is transversal to the plane $S+I+R=P_{\text {total }}$. Indeed, the tangent vector to the curve has the components 


$$
\begin{aligned}
T: \frac{\partial S}{\partial I} & =\frac{1}{2}\left(\beta+\frac{\beta^{2} I-2 \gamma}{\sqrt{\left(\beta^{2} I-4 \gamma\right) I}}\right), \\
\frac{\partial I}{\partial I} & =1, \\
\frac{\partial R}{\partial I} & =\frac{\beta}{\gamma} S-1
\end{aligned}
$$

the normal vector to the plane has the components $N=(1,1,1)$, and the scalar product $\langle T, N\rangle$ is different from zero.

\section{Epidemic Wind}

The geometric data of the world $[9,10]$ change the epidemic flow into an epidemic wind. This is a new idea that we are adding to the spread of infections.

So far, predictive mathematical models for epidemics were treated as flows. Now, we add a more complex idea, namely, to look at the evolution of an epidemic as a wind created ad hoc by the epidemic flow and the "geometry of the world." These are fundamental to understand the course of the epidemics and to plan effective control strategies for answering the question: how can we explain an exponentially growing number of patients all over the world who were diagnosed with COVID-19?

The time has come for us to treat the epidemics like winds (geometric dynamics and geodesic motion in a gyroscopic field of forces) $[9,10]$, producing chaotic dynamics. The geometric dynamics is generated by primordial data: flow and geometry of the space.
The infectious disease autonomous flow on $\mathbb{R}_{+}^{3}$ is

$$
\begin{aligned}
& \dot{S}(t)=-\beta S(t) I(t), \\
& \dot{I}(t)=(\beta S(t)-\gamma) I(t), \\
& \dot{R}(t)=\gamma I(t) .
\end{aligned}
$$

On the Riemannian manifold $\left(\mathbb{R}_{+}^{3}, \delta_{i j}\right)$, the flow and the metric determine the least squares autonomous Lagrangian

$$
\begin{aligned}
2 L= & (\dot{S}(t)+\beta S(t) I(t))^{2}+(\dot{I}(t)-(\beta S(t)-\gamma) I(t))^{2} \\
& +(\dot{R}(t)-\gamma I(t))^{2} .
\end{aligned}
$$

We attach an integral action

$$
C(S(\cdot), I(\cdot), R(\cdot))=\int_{t_{0}}^{t_{1}} L(S(t), I(t), R(t), \dot{S}(t), \dot{I}(t), \dot{R}(t)) \mathrm{d} t .
$$

A geometric dynamics $[9,10]$ appears, described by the Euler-Lagrange ODEs

$$
\begin{aligned}
& \frac{\partial L}{\partial S}-\frac{\mathrm{d}}{\mathrm{d} t} \frac{\partial L}{\partial \dot{S}}=0, \\
& \frac{\partial L}{\partial I}-\frac{\mathrm{d}}{\mathrm{d} t} \frac{\partial L}{\partial \dot{I}}=0 \\
& \frac{\partial L}{\partial R}-\frac{\mathrm{d}}{\mathrm{d} t} \frac{\partial L}{\partial \dot{R}}=0 .
\end{aligned}
$$

Explicitly, the epidemic wind is described by the secondorder differential system

$$
\begin{aligned}
& \beta I(t)(\dot{S}(t)-\dot{I}(t))+2 \beta(S(t)-\gamma) I(t)^{2}-\frac{\mathrm{d}}{\mathrm{d} t}(\dot{S}(t)+\beta S(t) I(t))=0, \\
& \beta S(t)(\dot{S}(t)-\dot{I}(t))+\gamma \dot{I}(t)-\gamma \dot{R}(t)+2 \beta^{2} S(t)^{2} I(t)^{2}-\beta \gamma S(t) I(t)+\gamma^{2} I(t) \\
& \quad-\frac{\mathrm{d}}{\mathrm{d} t}(\dot{I}(t)-\beta S(t) I(t)+\gamma I(t))=0, \\
& \frac{\mathrm{d}}{\mathrm{d} t}(\dot{R}(t)-\gamma I(t))=0 .
\end{aligned}
$$

Adding all three ODEs, we obtain an ODE whose last term is $-(\ddot{S}(t)+\ddot{I}(t)+\ddot{R}(t))$. Furthermore, the last secondorder ODE is equivalent to the first-order ODE

$$
\dot{R}(t)-\gamma I(t)=c_{1} .
$$

Theorem 5. The geometric dynamics (wind) represented by previous second-order ODEs is decomposable into the infectious disease flow and transversal to flow spiral trajectories.
Proof. We give the proof in generic coordinates. The subset of solutions corresponding to the initial values $x\left(t_{0}\right)=x_{0}, \dot{x}\left(t_{0}\right)=X\left(x\left(t_{0}\right)\right)$ are solutions reducible to solutions of the infectious disease flow. The subset of solutions corresponding to the initial values $x\left(t_{0}\right)=x_{0}, \dot{x}$ $\left(t_{0}\right)=W \neq \lambda X\left(x\left(t_{0}\right)\right), \lambda>0$, are transversal to the solutions of the infectious disease flow. The converse is also true.

Based on the existence and uniqueness theorem, each solution $x=x(t)$ of any second-order prolongation of the 
first-order ODE system has the property: $\dot{x}\left(t_{0}\right)=X\left(x\left(t_{0}\right)\right)$ implies $\dot{x}(t)=X(x(t)), \forall t \in I$.

In generic coordinates, the attached Hamiltonian to the wind is

$$
H=\frac{1}{2}\langle\dot{x}-X(x), \dot{x}+X(x)\rangle=\frac{1}{2}\|\dot{x}\|^{2}-f(x),
$$

where $f(x)=(1 / 2)\|X(x)\|^{2}$ (kinetic energy). The maximal solutions of infectious disease wind are split into three categories of curves: (1) curves characterized by $H=0$ (flow trajectories); (2) curves satisfying $H=$ const $>0$; (3) curves characterized by $H=$ const $<0$. The transversal curves in category (2) can have the images throughout, but the curves in category (3) have the images only in the set $f(x) \geq-H$.

The solutions of infectious disease wind are highly sensitive to initial conditions. In other words, small differences in initial conditions, such as those due to rounding errors in numerical computation, can yield widely diverging outcomes for infectious disease wind, rendering long-term prediction of its behavior impossible in general. A single field line and an infinity of transverse curves start from a fixed point.

In a flow, the starting point is fixed, but the endpoint is the one that results. In geometric dynamics, the initial conditions are in the form of point-direction point-endpoint The solution we find optimizes the objective function of the smallest squares (the best approximation of flow in the sense of a convenient Riemannian metric). Small disturbances of the initial direction or of the endpoint can produce dramatic changes in the solution, which highlight the complexity of the problem.

Remark 3. In our sense, any wind is strongly dependent on the Riemannian manifold $M, g_{i j}(x)$. The best selection of the Riemannian manifold adapted to infectious disease wind is after constant curvature: (1) curvature 0, Euclidean manifold $\mathbb{R}^{3}, g_{i j}(x)=\delta_{i j}$, used in the previous explanations; (2) curvature -1 , hyperbolic manifold $\left(\mathbb{H}^{3}, g_{i j}(x)=\left(\delta_{i j} / x^{32}\right)\right) ; \quad(2) \quad$ curvature 1 , sphere $S^{3}=\left\{x=\left(x^{1}, x^{2}, x^{3}, x^{4}\right) \in \mathbb{R}^{4} \mid\|x\|^{2}=1\right\}$, with the metric induced by the Euclidean metric on $\mathbb{R}^{4}$.

\section{Stochastic Connectivity}

Stochastic differential equations are widely used to model epidemic infections, molecular dynamics, biophysical dynamics, climate dynamics, engineering systems, and so on, under random fluctuations.

Let us write the flow in Pfaff terminology and let us replace the parameter $\beta$ by a control $u(t)$. We use $W^{a}(t), t \geq 0, a=1,2,3$, as independent Wiener processes. Starting from the (nonlinear control system) infectious disease Cauchy problem

$$
\begin{aligned}
\mathrm{d} S(t) & =-u(t) S(t) I(t) \mathrm{d} t, \\
\mathrm{~d} I(t) & =(u(t) S(t)-\gamma) I(t) \mathrm{d} t, \\
\mathrm{~d} R(t) & =\gamma I(t) \mathrm{d} t, \\
S(0) & =S_{0}, \\
I(0) & =I_{0}, \\
R(0) & =R_{0}, \\
S_{0}+I_{0}+R_{0} & =P_{\text {total }},
\end{aligned}
$$

on $\mathbb{R}_{+}^{3}$, a stochastic perturbation is defined by stochastic differential equation system,

$$
\begin{aligned}
\mathrm{d} S(t) & =-u(t) S(t) I(t) \mathrm{d} t+\sigma_{a}^{1}(t, S(t), I(t), R(t), u(t)) \mathrm{d} W^{a}(t), \\
\mathrm{d} I(t) & =(u(t) S(t)-\gamma) I(t) \mathrm{d} t+\sigma_{a}^{2}(t, S(t), I(t), R(t), u(t)) \mathrm{d} W^{a}(t), \\
\mathrm{d} R(t) & =\gamma I(t) d t-\sigma_{a}^{3}(t, S(t), I(t), R(t), u(t)) \mathrm{d} W^{a}(t), \\
S(0) & =S_{0}, \\
I(0) & =I_{0}, \\
R(0) & =R_{0}, \\
S_{0}+I_{0}+R_{0} & =P_{\text {total }},
\end{aligned}
$$

where the functions $u(t) S(t) I(t), u(t) S(t)$ $I(t)-\gamma I(t), \gamma I(t)$ are drift coefficients and $\sigma_{a}^{i}(t, S(t), I(t), R(t), u(t)), i=1,2,3 ; a=1, \ldots, d$, are diffusion coefficients.

If $S_{0}+I_{0}+R_{0}=P_{\text {total }}$ and $\sigma_{a}^{1}+\sigma_{a}^{2}=\sigma_{a}^{3}$, then the stochastic perturbation satisfies $\mathrm{d} S(t)+\mathrm{d} I(t)+\mathrm{d} R(t)=0$; that is, we have again a first integral $S(t)+I(t)+R(t)=P_{\text {total }}$.

Suppose that the control $u(t)$ is piecewise smooth and has values in a bounded and closed set $U=[\delta, m] \subset(0, \infty)$, where $m$ will be selected at the end of this section by an extremum problem, determining the optimal striking time.
The set of such controls, denoted by $\mathcal{U}$, is called the set of admissible controls.

We explore how stochastic noise can be used to find connectivity properties generated by the underlying deterministic infectious disease dynamics and randomness.

Definition 1. A strong solution $c(t)=(S(t), I(t), R(t))$ of this stochastic differential system with the initial condition $\left(S_{0}, I_{0}, R_{0}\right)$ is an adapted continuous process, such that, for all $t \geq 0$, it satisfies the stochastic integral system 


$$
\begin{aligned}
S(t)-S_{0} & =-\int_{0}^{t} u(s) S(s) I(s) \mathrm{d} s+\int_{0}^{t} \sigma_{a}^{1}(s, S(s), I(s), R(s), u(s)) \mathrm{d} W^{a}(s), \\
I(t)-I_{0} & =\int_{0}^{t} u(s) S(s)-\gamma I(s) \mathrm{d} s+\int_{0}^{t} \sigma_{a}^{2}(s, S(s), I(s), R(s), u(s)) \mathrm{d} W^{a}(s), \\
R(t)-R_{0} & =\int_{0}^{t} \gamma I(s) \mathrm{d} s-\int_{0}^{t} \sigma_{a}^{3}(s, S(s), I(s), R(s), u(s)) \mathrm{d} W^{a}(s), a . s .
\end{aligned}
$$

However, there is a number of subtle points involved: first, the existence of the integrals requires some degree of regularity on $c(t)=(S(t), I(t), R(t))$ and on the functions $u(t), \sigma=\left(\sigma_{a}^{i}\right)$ (matrix); in particular, it must be the case that, for all $t \geq 0$, with probability one, $\int_{0}^{t}\|\sigma(s)\|^{2} \mathrm{~d} s<\infty$.

Second, the solution is required to exist for all $t<\infty$ with probability one.

Properties of Itô integral are that, for all $G, H \in \mathbb{L}^{2}(0, T)$, we have

$$
\begin{aligned}
\mathbb{E}\left(\int_{0}^{T} G \mathrm{~d} W\right) & =0 \\
\mathbb{E}\left(\left(\int_{0}^{T} G \mathrm{~d} W\right)^{2}\right) & =\mathbb{E}\left(\int_{0}^{T} G^{2} \mathrm{~d} t\right), \\
\mathbb{E}\left(\left(\int_{0}^{T} G \mathrm{~d} W\right)\left(\int_{0}^{T} H \mathrm{~d} W\right)\right) & =\mathbb{E}\left(\int_{0}^{T} G H \mathrm{~d} t\right) .
\end{aligned}
$$

Definition 2. A weak solution $c(t)=(S(t), I(t), R(t))$ of the SDE with the initial condition $\left(S_{0}, I_{0}, R_{0}\right)$ is a continuous stochastic process defined on some probability space
$(\Omega, \mathfrak{F}, P)$, such that, for some Wiener processes $W^{a}(t)$ and some admissible filtration $\mathfrak{F}$, the process $c(t)$ is adapted and satisfies the associated stochastic integral system.

The drift coefficients are uniformly Lipschitz functions. The basic result, due to Itô, is that, for uniformly Lipschitz functions $\sigma$, the SDE has strong solutions and that, for each initial value $c(0)=(S(0), I(0), R(0))$, the solution is unique.

Without loss of generality, we consider that the amplitudes of error are constants; that is, $\sigma_{a}^{i}(t, S(t), I(t), R(t), u(t))=\sigma_{a}^{i}$.

Definition 3. Let $u(t)=u(S(t), I(t), R(t))$ be a feedback bounded control. A stochastic process $c(t)=(S(t), I(t), R(t))$, which satisfies the SDE system, is called an admissible stochastic process.

Theorem 6. Let $P=\left(S_{P}, I_{P}, R_{P}\right)$ and $Q=\left(S_{Q}, I_{Q}, R_{Q}\right)$ be two points in the set $[0,1]^{3} \subset \mathbb{R}_{+}^{3}$. Denote $D(Q, r)$ as the Euclidean sphere of radius $r$, centered at $Q$. Then, for any $\varepsilon>0$ and $r>0$, there exists a striking time $t<\infty$ and an admissible stochastic process $c(t)$, satisfying the boundary conditions

such that

$$
(S(0), I(0), R(0))=\left(S_{P}, I_{P}, R_{P}\right),(\mathbb{E}[S(t)], \mathbb{E}[I(t)], \mathbb{E}[R(t)])=\left(S_{Q}, I_{Q}, R_{Q}\right),
$$

$$
\mathbb{P}(c(t) \in D(Q, r)) \geq 1-\varepsilon .
$$

Proof. We start with the nonnegative random variable $\|c(t)-Q\|^{2}$. Markov inequality shows that

$$
\mathbb{P}\left(\|c(t)-Q\|^{2} \geq r^{2}\right) \leq \frac{1}{r^{2}} \mathbb{E}\left[\|c(t)-Q\|^{2}\right],
$$

or, equivalently,

$$
\mathbb{P}\left(\|c(t)-Q\|^{2} \leq r^{2}\right) \geq 1-\frac{1}{r^{2}} \mathbb{E}\left[\|c(t)-Q\|^{2}\right] .
$$

The stochastic integral variant of the previous SDE system is

$$
\begin{aligned}
& S(t)=-\int_{0}^{t} u(s) S(s) I(s) \mathrm{d} s+\sigma_{a}^{1} W^{a}(t)+S_{P}, \\
& I(t)=\int_{0}^{t}(u(s) S(s)-\gamma) I(s) \mathrm{d} s+\sigma_{a}^{2} W^{a}(t)+I_{P}, \\
& R(t)=\int_{0}^{t} \gamma I(s) \mathrm{d} s-\sigma_{a}^{3} W^{a}(t)+R_{P} .
\end{aligned}
$$

It is well-known that the expectation $\mathbb{E}$ has the properties

$$
\begin{aligned}
\mathbb{E}\left[W^{a}(t)\right] & =0, \\
\mathbb{E}\left[\left(W^{a}(t)\right)^{2}\right] & =t \\
\mathbb{E}\left[W^{a}(t) W^{b}(t)\right] & =0, \quad a \neq b .
\end{aligned}
$$

If two random variables $X, Y: \Omega \longrightarrow \mathbb{R}$ are independent, then $\mathbb{E}[X Y]=\mathbb{E}[X] \mathbb{E}[Y]$, provided that $\mathbb{E}[|X|]<\infty$ and $\mathbb{E}[|Y|]<\infty$.

The boundary conditions produce 


$$
\begin{aligned}
& \mathbb{E}[S(t)]=S_{Q} \Rightarrow \int_{0}^{t} \mathbb{E}[-u(s) S(s) I(s)] \mathrm{d} s+S_{P}=S_{Q}, \\
& \mathbb{E}[I(t)]=I_{Q} \Rightarrow \int_{0}^{t} \mathbb{E}[(u(s) S(s)-\gamma) I(s)] \mathrm{d} s+I_{P}=I_{Q}, \\
& \mathbb{E}[R(t)]=R_{Q} \Rightarrow \int_{0}^{t} \mathbb{E}[\gamma I(s)] \mathrm{d} s+R_{P}=R_{Q} .
\end{aligned}
$$

Moreover, $S_{P}+I_{P}+R_{P}=1$ implies $S_{Q}+I_{Q}+R_{Q}=1$.

We use the advantages of Itô integral and the independence of Wiener processes $W^{a}(t)$. We find

$$
\begin{aligned}
\mathbb{E}\left[\|c(t)-Q\|^{2}\right]= & \mathbb{E}\left[\left(S(t)-S_{Q}\right)^{2}\right]+\mathbb{E}\left[\left(I(t)-I_{Q}\right)^{2}\right]+\mathbb{E}\left[\left(R(t)-R_{Q}\right)^{2}\right] \\
= & \mathbb{E}\left[\left(-\int_{0}^{t} u(s) S(s) I(s) \mathrm{d} s+\sigma_{a}^{1} W^{a}(t)+S_{P}-S_{Q}\right)^{2}\right] \\
& +\mathbb{E}\left[\left(\int_{0}^{t}(u(s) S(s)-\gamma) I(s) \mathrm{d} s+\sigma_{a}^{2} W^{a}(t)+I_{P}-I_{Q}\right)^{2}\right] \\
& +\mathbb{E}\left[\left(\int_{0}^{t} \gamma I(s) \mathrm{d} s-\sigma_{a}^{3} W^{a}(t)+R_{P}-R_{Q}\right)^{2}\right] \\
= & \mathbb{E}\left[\int_{0}^{t} u(s) S(s) I(s) \mathrm{d} s \int_{0}^{t} u(s) S(s) I(s) u(s) \mathrm{d} s+\left(\sigma_{a}^{1} W^{a}(t)\right)^{2}+\left(S_{P}-S_{Q}\right)^{2}\right. \\
& \left.-2\left(\int_{0}^{t} u(s) S(s) I(s) \mathrm{d} s\right) \sigma_{a}^{1} W^{a}(t)-2\left(\int_{0}^{t} u(s) S(s) I(s) \mathrm{d} s\right)\left(S_{P}-S_{Q}\right)+2 \sigma_{a}^{1} W^{a}(t)\left(S_{P}-S_{Q}\right)\right] \\
& +\mathbb{E} \int_{0}^{t}(u(s) S(s)-\gamma) I(s) \mathrm{d} s \int_{0}^{t}(u(s) S(s)-\gamma) I(s) \mathrm{d} s \\
& +\left(\sigma_{a}^{2} W^{a}(t)\right)^{2}+\left(I_{P}-I_{Q}\right)^{2}+2\left(\int_{0}^{t}(u(s) S(s)-\gamma) I(s) \mathrm{d} s\right) \sigma_{a}^{2} W^{a}(t) \\
& +2\left(\int_{0}^{t}(u(s) S(s)-\gamma) I(s) \mathrm{d} s\right)\left(I_{P}-I_{Q}\right)+2 \sigma_{a}^{2} W^{a}(t)\left(I_{P}-I_{Q}\right) \\
& +\mathbb{E}\left[\int_{0}^{t} \gamma I(s) \mathrm{d} s \int_{0}^{t} \gamma I(s) \mathrm{d} s+\left(\sigma_{a}^{3} W^{a}(t)\right)^{2}+\left(R_{P}-R_{Q}\right)^{2}\right. \\
& \left.-2\left(\int_{0}^{t} \gamma I(s) \mathrm{d} s\right) \sigma_{a}^{3} W^{a}(t)-2\left(\int_{0}^{t} \gamma I(s) \mathrm{d} s\right)\left(R_{P}-R_{Q}\right)+2 \sigma_{a}^{3} W^{a}(t)\left(R_{P}-R_{Q}\right)\right] .
\end{aligned}
$$

This last expression is reduced to

$$
\begin{aligned}
& \mathbb{E}\left[\int_{0}^{t} u(s) S(s) I(s) \mathrm{d} s \int_{0}^{t} u(s) S(s) I(s) \mathrm{d} s\right]+t \sum_{a} \sigma_{a}^{1} \sigma_{a}^{1}-\left(S_{P}-S_{Q}\right)^{2} \\
& +\mathbb{E}\left[\int_{0}^{t}(u(s) S(s)-\gamma) I(s) \mathrm{d} s \int_{0}^{t}(u(s) S(s)-\gamma) I(s) \mathrm{d} s\right]+t \sum_{a} \sigma_{a}^{2} \sigma_{a}^{2}-\left(I_{P}-I_{Q}\right)^{2} \\
& +\mathbb{E}\left[\int_{0}^{t} \gamma I(s) \mathrm{d} s \int_{0}^{t} \gamma I(s) \mathrm{d} s\right]+t \sum_{a} \sigma_{a}^{3} \sigma_{a}^{3}-\left(R_{P}-R_{Q}\right)^{2} \\
& =\mathbb{E}\left[\left(\int_{0}^{t} u(s) S(s) I(s) \mathrm{d} s\right)^{2}\right]+\mathbb{E}\left[\left(\int_{0}^{t}(u(s) S(s)-\gamma) I(s) \mathrm{d} s\right)^{2}\right] \\
& +\mathbb{E}\left[\left(\int_{0}^{t} \gamma I(s) \mathrm{d} s\right)^{2}\right]+t \delta_{i j} \sum_{a} \sigma_{a}^{i} \sigma_{a}^{j}-\|P-Q\|^{2} .
\end{aligned}
$$


On the other hand, the Cauchy-Schwarz inequalities

$$
\begin{gathered}
\left(\int_{0}^{t} u(s) S(s) I(s) \mathrm{d} s\right)^{2} \leq t \int_{0}^{t}(u(s) S(s) I(s))^{2} \mathrm{~d} s \\
\left(\int_{0}^{t}(u(s) S(s)-\gamma) I(s) \mathrm{d} s\right)^{2} \leq t \int_{0}^{t}(u(s) S(s)-\gamma)^{2} I^{2}(s) \mathrm{d} s, \\
\left(\int_{0}^{t} \gamma I(s) \mathrm{d} s\right)^{2} \leq t \int_{0}^{t}(\gamma I(s))^{2} \mathrm{~d} s,
\end{gathered}
$$

the properties of expectation, and the condition $u(t) \leq m$ produce the estimations

$$
\begin{gathered}
\mathbb{E}\left[\left(\int_{0}^{t} u(s) S(s) I(s) \mathrm{d} s\right)^{2}\right] \leq m^{2} S_{Q}^{2} I_{Q}^{2} t^{2}, \\
\mathbb{E}\left[\left(\int_{0}^{t}(u(s) S(s)-\gamma) I(s) \mathrm{d} s\right)^{2}\right] \leq\left(m S_{Q}-\gamma\right)^{2} I_{Q}^{2} t^{2}, \\
\mathbb{E}\left[\left(\int_{0}^{t} \gamma I(s) \mathrm{d} s\right)^{2}\right] \leq \gamma^{2} I_{Q}^{2} t^{2} .
\end{gathered}
$$

Denoting

$$
\begin{aligned}
k_{1}^{2} & =m^{2} S_{Q}^{2} I_{Q}^{2}, \\
k_{2}^{2} & =\left(m S_{Q}-\gamma\right)^{2} I_{Q}^{2}, \\
k_{3}^{2} & =\gamma^{2} I_{Q}^{2}, \\
\|\sigma\|^{2} & =\delta_{i j} \sum_{a} \sigma_{a}^{i} \sigma_{a}^{j},
\end{aligned}
$$

we find

$$
\mathbb{E}\left[\|c(t)-Q\|^{2}\right] \leq\left(k_{1}^{2}+k_{2}^{2}+k_{3}^{2}\right) t^{2}+\|\sigma\|^{2} t-\|P-Q\|^{2} .
$$

For any $\epsilon>0$ and $r>0$, the equation

$$
\left(k_{1}^{2}+k_{2}^{2}+k_{3}^{2}\right) t^{2}+\|\sigma\|^{2} t-\|P-Q\|^{2}-\varepsilon r^{2}=0,
$$

has a strictly positive solution

$$
t_{+}=\frac{-\|\sigma\|^{2}+\sqrt{\|\sigma\|^{4}+4\left(k_{1}^{2}+k_{2}^{2}+k_{3}^{2}\right) \Lambda^{2}}}{2\left(k_{1}^{2}+k_{2}^{2}+k_{3}^{2}\right)}
$$

where $\Lambda^{2}=\|P-Q\|^{2}+\varepsilon r^{2}$.

Denote $\|\sigma\|=c, k_{1}^{2}=m^{2} a^{2}$, and $k_{2}^{2}=(m S-g)^{2} b^{2}$. Using Maple routines, we find $t_{+e}=\operatorname{extrema}_{m} t_{+}$:

(i) extrema-

$$
\begin{aligned}
& \left(\left(-c^{2}+\operatorname{sqrt}\left(c^{4}+\left(4 * \left(m^{2} * a^{2}+(S * m-g)^{2}\right.\right.\right.\right.\right. \\
& \left.\left.\left.\left.* b^{2}+k 3^{2}\right)\right) * A\right)\right) /\left(2 * \left(m^{2} * a^{2}+\right.\right. \\
& \left.\left.\left.(S * m-g)^{2} * b^{2}+k 3^{2}\right)\right),, m\right)
\end{aligned}
$$

Answer:

$$
\frac{1}{2} \frac{\left(-c^{2}+\sqrt{\left(\left(S^{2} b^{2} c^{4}+4 A S^{2} b^{2} k 3^{2}+4 A a^{2} b^{2} g^{2}+a^{2} c^{4}+4 A a^{2} k 3^{2}\right) /\left(S^{2} b^{2}+a^{2}\right)\right)}\right)\left(S^{2} b^{2}+a^{2}\right)}{S^{2} b^{2} k 3^{2}+a^{2} b^{2} g^{2}+a^{2} k 3^{2}} .
$$

For this optimal solution $t_{+e}$, we can write

$$
\mathbb{E}\left[\left\|c\left(t_{+e}\right)-Q\right\|^{2}\right] \leq \varepsilon r^{2} \Leftrightarrow 1-\frac{1}{r^{2}} \mathbb{E}\left[\left\|c\left(t_{+e}\right)-Q\right\|^{2}\right] \geq 1-\varepsilon,
$$

that is, $\mathbb{P}\left(c\left(t_{+e}\right) \in D(Q, r)\right) \geq 1-\varepsilon$.

The previous theorem confirms that almost sure any two points in the "world" are joined by a stochastic curve. So, contamination can get anywhere from anywhere.

\section{Conclusions}

The most difficult thing for an epidemiological model is to predict human behavior. Infected population size estimation is a common problem in any epidemic analysis and it is the most important aspect for planning appropriate care and prevention policies. We underline that a model, even complex, is a simplification of reality, since a lot of other variables are still not completely known. An interactive approach with Maple can clarify some of these issues.

The flow models and even differential inclusion models are varied, often confusing to interpret, and are not crystal balls, especially because the ideal data are not yet available. But they are a large part of what government leaders use to make decisions, influencing how resources are allocated to health care facilities and how social distancing orders are issued to the public.

The three points of view in this paper, infectious disease flow, infectious disease wind, and stochastic connectivity, can input the biology of the virus and give positive answers to the following questions: how does it spread, how quickly 
does it infect, how quickly does it lead to symptoms, and how quickly does it replicate to a level where it can jump from person to person?

The infectious disease wind is determined by the flow and by the geometry of space (created by nature or by human activities, conscious or not). All (the flow, the geometry, and the least squares technique) generate gyroscopic forces that lead to spiraling (uncontrolled) evolutions. The Riemannian structure can generate also chaos in geometric dynamics (sensitive dependence on initial conditions, and three or more dimensions). The experts in the fields of surveillance, epidemiology, and prevention of communicable disease spread, infection preventionists, must understand that infectious diseases modeled as wind and stochastic modeling of geometric structures are responsible for reading the infectious disease disasters.

\section{Data Availability}

The underlying data supporting the results of our study can be found through Prof. Emeritus Dr. Constantin Udriste.

\section{Conflicts of Interest}

The authors declare that they have no conflicts of interest.

\section{Acknowledgments}

The authors would like to thank Prof. Dr. Ovidiu Calin, Eastern Michigan University, for valuable remarks on our manuscript, and Miss Lecturer Dr. Oana-Maria Pastae, "Constantin Brancusi" University of Tg-Jiu, for checking the use of the English language in the paper.

\section{References}

[1] O. Calin and C. Udriste, Geometric Modeling in Probability and Statistics, Springer, Berlin, Germany, 2014.

[2] O. Calin, C. Udriste, and I. Tevy, "Stochastic sub-riemannian geodesics on grushin distribution," Balkan Journal of Geometry and Its Applications, vol. 19, no. 2, pp. 37-49, 2014.

[3] A. Simha, R. V. Prasad, and S. Narayana, "A simple stochastic sir model for COVID-19 infection dynamics for karnataka - learning from europe," 2020, http://arxiv.org/abs/2003. 11920v1.

[4] T. Turcanu and C. Udriste, "Stochastic connectivity on a almost-Riemannian structure induced by symmetric polynomials," Scientific Bulletin-University Politehnica of Bucharest, vol. 81, no. 2, pp. 17-22, 2019.

[5] G. Giordano, F. Blanchini, R. Bruno et al., "Modelling the COVID-19 epidemic and implementation of population-wide interventions in Italy," Letters, vol. 14, 2020.

[6] M. W. Hirsch, S. Smale, and R. L. Devaney, Differential Equations, Dynamical Systems and Introduction to Chaos, Academic Press, Cambridge, UK, 2004.

[7] Y. Maki and H. Hideo, "Infectious disease spread analysis using stochastic differential equations for SIR model," in Proceedings of the 4th International Conference on Intelligent Systems, Modelling and Simulation, IEEE, Bangkok, Thailand, 2013.
[8] J.-P. Aubin and A. Cellina, Differential Inclusions (Set-Valued Maps and Viability Theory), Springer-Verlag, Berlin, Germany, 1984.

[9] C. Udriste, Geometric Dynamics, Kluwer Academic Publishers, Amsterdam, Netherlands, 2000.

[10] C. Udriste and I. Tevy, "Geometric dynamics on Riemannian manifolds," Mathematics, vol. 8, no. 79, pp. 1-14, 2020.

[11] C. Udriste, O. Dogaru, M. Ferrara, and I. Tevy, "Nonholonomic optimization," in Recent Advances in Optimization. Lecture Notes in Economics and Mathematical Systems, A. Seeger, Ed., vol. vol 563, pp. 119-132, Springer, Berlin, Germany, 2006.

[12] C. Udriste, M. Constantinescu, I. Tevy, and O. Dogaru, "Dualities in nonholonomic optimization," Mathematics and Computer Science, vol. 54, no. 2, pp. 149-166, 2016.

[13] G. Vranceanu, "Sur les spaces non holonomes," Comptes rendus de l'Académie des Sciences, vol. 183, pp. 852-854, 1926. 